\title{
Movimentos da Estrutura a Termo e Critérios de Minimização do Erro de Previsão em um Modelo Paramétrico Exponencial* $^{*}$
}

\author{
Caio Almeida ${ }^{\dagger}$, Romeu Gomes ${ }^{\ddagger}$, André Leite ${ }^{\S}$, José Vicente $₫$
}

\author{
Conteúdo: 1. Introdução; 2. Dados; 3. O Modelo DL; 4. Análise dos Resultados; 5. Conclusão; \\ A. Figuras. \\ Palavras-chave: Modelos Paramétricos de Estrutura a Termo; Componentes Principais; Previsão \\ da Curva de Juros. \\ Códigos JEL: $\quad$ G1; E4; C5.
}

Neste artigo, nós estudamos como diferentes escolhas dos loadings afetam a previsão do modelo exponencial de estrutura a termo proposto por Diebold e Li (2006). Os loadings são definidos através de um parâmetro específico lambda que controla tanto a velocidade de decaimento da inclinação como o máximo da curvatura. Em particular, adotando uma base de dados formada por taxas brasileiras de DI futuro, nós analisamos quatro regras de escolha que dependem de métricas que minimizam erros de previsão para diferentes horizontes de previsão. Nós concluímos que a regra ótima muda de acordo com a região dos vencimentos dos DI futuro e com o horizonte de previsão. Este fato indica que a escolha de como os movimentos são parametrizados no modelo Diebold e Li (2006), deve ser feita com cuidado, adaptada para aplicação particular.

In this paper, we study how different choices of loadings affect forecasting in the exponential term structure model proposed by Diebold e Li (2006). The loadings are defined through a specific parameter lambda which controls both the decaying speed of the slope as well as the maximum of the curvature factors. In particular, adopting a database including Brazilian fixed income future

\footnotetext{
*As opiniões expressas neste artigo são dos autores e não necessariamente refletem aquelas do Banco Central do Brasil. 0 primeiro autor agradece o suporte financeiro dado pelo CNPq.

${ }^{\dagger}$ EPGE, Fundação Getulio Vargas. E-mail: calmeida@fgv . br

¥Banco Central do Brasil. E-mail: romeu. gomes@bcb. gov . br

$\S$ Banco Central do Brasil. E-mail: andreluis. leite@bcb.gov.br

I Banco Central do Brasil e Faculdades Ibmec-RJ. Autor correspondente. E-mail: jose.valentim@bcb.gov .br
} 
contracts (ID future), we analyze four different rules of choices depending on metrics that minimize forecasting errors, for different forecasting horizons. We conclude that the optimal rule changes for different regions of ID future maturities/different forecasting horizons. This fact indicates that the choice of how movements will be parameterized in the Diebold e Li (2006), model should be done with care, tailored for each particular application.

\section{INTRODUÇÃO}

Previsão da Estrutura a Termo de Taxa de Juros (ETTJ) tem enorme importância tanto para os tomadores de decisões de política monetária como para os participantes do mercado de renda fixa. Neste artigo, nós analisamos a capacidade preditiva do modelo de Diebold e Li (2006) (modelo DL daqui em diante) considerando quatro modos diferentes de estimação do parâmetro do modelo que controla a inclinação e a curvatura da curva de juros. Os resultados mostram que dependendo do horizonte de previsão e da taxa que se têm em foco, estratégias de estimação diferentes desse parâmetro devem ser empregadas.

Apesar da relevância do assunto e do grande progresso na modelagem da curva de juros observado nos últimos anos, pouca atenção foi dada na literatura acadêmica à questão de previsão da ETTJ. Basicamente, estudos da ETTJ concentram-se em duas classes de modelos, comumente conhecidos como estatísticos e de equilíbrio (veja Varga (2005)). No primeiro grupo, a ETTJ é construída via um procedimento de interpolação e previsões são feitas usando modelos de séries temporais. No segundo grupo, os modelos incorporam argumentos de equilíbrio, tais como não-arbitragem, para analisar a curva de juros. A estimação dos modelos estatísticos é em geral menos complexa. Porém o uso de técnicas econométricas tradicionais pode apresentar fraco desempenho preditivo das taxas futuras (veja, por exemplo, Diebold e Li (2006) e Lima et alii (2006). Por outro lado, embora fundamentados economicamente, os modelos de equilíbrio também não garantem sucesso na previsão de taxas futuras, como bem demonstra Duffee (2002)).

Recentemente, em um artigo publicado no Journal of Econometrics, os pesquisadores Francis Diebold e Canlin Li (Diebold e Li, 2006) apresentaram uma técnica para previsão da ETTJ americana com resultados significativamente melhores que diversos modelos tradicionais de séries temporais. O modelo DL é uma variante do modelo puramente estatístico de Nelson e Siegel (1987) (modelo NS daqui em diante). ${ }^{1}$ No modelo DL, uma série temporal de três fatores estocásticos (associados com o nível, inclinação e curvatura da curva de juros) são estimados por mínimos quadrados a partir de uma amostra de taxas à vista. Em seguida faz-se a previsão fora da amostra desses fatores usando um processo autoregressivo de ordem 1 (AR(1)).

Muito provavelmente o sucesso do modelo DL deve-se não somente aos bons resultados obtidos mas também a facilidade de implementação da técnica. Conseqüentemente, embora recente, o modelo DL já é objeto de diversos estudos e extensões. Vicente e Tabak (2008) comparam o modelo afim gaussiano ${ }^{2}$ com o modelo DL e concluem que este último tem poder de previsão ligeiramente superior ao primeiro para a curva brasileira. Almeida et alii (2007) propõem uma versão dinâmica do modelo de Svensson (1994) para ajustar a curva de juros e obtêm resultados superiores aos do modelo DL. Laurini e Hotta (2007) mostram que extensões bayesianas do modelo DL aumentam significativamente a capacidade preditiva. Koopman et alii (2008) apresentam evidências de que loadings $s^{3}$ e volatilidades variantes no

\footnotetext{
${ }^{1}$ Filipovic e Sharef (2004) mostram como modificar o modelo paramétrico NS de modo a impedir a ocorrência de oportunidades de arbitragem.

${ }^{2}$ Veja Dai e Singleton (2000) para uma descrição detalhada dos modelos afim.

${ }^{3}$ Os loadings de um modelo paramétrico correspondem aos autovetores de uma rotação da matriz de covariância das taxas (veja Almeida et alii (2003)).
} 
tempo agregam um resultado positivo às previsões do modelo DL.

Também não tardaram a surgir na literatura trabalhos que testam a robustez do modelo DL. Varga (2007), usando uma base de dados de contratos DI futuro, mostra que o modelo DL não reproduz os bons resultados para a ETTJ brasileira. De Pooter (2007) avalia o desempenho do modelo DL em subamostras e conclui que a capacidade preditiva do modelo DL tem forte dependência com a base utilizada. Já Huse (2007) acrescenta que o modelo DL apresenta problemas de previsão em momentos de crise. Como alternativa, ele propõe uma modificação do modelo DL que inclui variáveis macroeconômicas para sanar esses problemas.

No entanto, até onde vai nosso conhecimento, não existe na literatura trabalhos que avaliam a robustez do modelo DL em termos da especificação dos loadings dos três movimentos da curva. ${ }^{4}$ No modelo DL, os loadings dos fatores dependem de um parâmetro que controla o ponto de máximo da curvatura e a velocidade de decaimento da inclinação. ${ }^{5}$ Valores diferentes do decaimento fornecem séries temporais diferentes para os fatores estocásticos e portanto previsões distintas. Diebold e Li (2006) sugerem fixar o parâmetro de decaimento de tal sorte que o máximo teórico da curvatura coincida com o máximo da curvatura extraída da amostra via análise de componentes principais. Embora de forte apelo intuitivo essa abordagem não exclui outras possibilidades igualmente coerentes.

Neste trabalho nós analisamos o desempenho em termos de previsão fora da amostra de quatro novas regras de escolha do parâmetro de decaimento. A base de dados do estudo consiste de uma série diária de taxas de juros à vista do mercado brasileiro. Na primeira regra o parâmetro de decaimento é determinado de modo a minimizar a soma dos quadrados dos erros de ajuste dentro da amostra. Nas outras três regras os dados dentro da amostra foram divididos em duas sub-bases. Em seguida escolhem-se os valores para o parâmetro de decaimento que minimizam o erro médio quadrático total das previsões diárias da ETTJ na segunda sub-base para um horizonte de previsão de 1 dia, 1 semana (5 dias úteis) e 1 mês (21 dias úteis). Por fim nós comparamos o poder de previsão para esses mesmos três horizontes temporais de cada uma das técnicas usando uma base de taxas fora da amostra. Em média, a regra ótima de escolha do parâmetro de decaimento para previsão fora da amostra em cada horizonte considerado coincide com o mesmo horizonte de previsão usado no ajuste na segunda sub-base dentro da amostra. Por exemplo, para fazer previsões 1 mês a frente fora da amostra, o parâmetro de decaimento que minimiza os erros de previsão 1 mês a frente na segunda sub-base dentro da amostra é, em média, superior aos demais. No entanto, dependendo da taxa de que prazo se deseja prever, essa conclusão pode não ser verdadeira. Esse resultado demonstra que as previsões do modelo DL são sensíveis à forma como se estima o parâmetro de controle da curvatura. Essa falta de robustez pode estar relacionada com a dificuldade da combinação de modelos paramétricos com processos AR(1) em capturar corretamente o prêmio de risco das taxas forward, conforme apontado por Almeida e Vicente (2008).

O restante do artigo é organizado como se segue. A Seção 2 apresenta uma análise descritiva dos dados usados neste estudo. A Seção 3 descreve o modelo DL e os quatro métodos de escolha do parâmetro de decaimento. Na Seção 4 é feita uma análise dos resultados obtidos. A Seção 5 conclui o trabalho.

\section{DADOS}

Os resultados descritos neste trabalho foram obtidos a partir de uma base de dados diários do mercado futuro de taxa média de depósitos interfinanceiros (DI) de um dia da BM\&F (Bolsa de Mercadorias

\footnotetext{
${ }^{4}$ Apesar de trabalharem com um especificação alternativa para os loadings, Koopman et alii (2008) modificam a parsimônia do modelo DL uma vez que consideram loadings variantes no tempo. Ao contrário, nós testamos diferentes formas para os loadings, mas mantendo a invariabilidade temporal dos mesmos, conforme proposto por Diebold e Li (2006).

${ }^{5}$ Nos referiremos a esse parâmetro como parâmetro de decaimento ou parãmetro de curvatura.
} 
$\&$ Futuros), ou mais resumidamente, DI futuro de 1 dia. O período de estudo engloba os pregões de 04/11/2004 a 28/12/2006 totalizando 534 dias de saques. ${ }^{6}$

No mercado futuro de DI de 1 dia da BM\&F é negociada a taxa de juros efetiva anual, base 252 dias úteis, até o vencimento do contrato, definida pela acumulação das taxas diárias de $\mathrm{DI}^{7}$ no período compreendido entre a data de negociação e o último dia de negociação do contrato. ${ }^{8}$ Geralmente, o mercado caracteriza cada contrato DI futuro pela data de vencimento.

Com a finalidade de eliminar dados oriundos de contratos pouco líquidos e que portanto poderiam conter informação distorcida, nós eliminamos em cada dia de pregão os vencimentos com volume negociado inferior a 500 contratos. A cada dia o prazo para o vencimento de um contrato DI futuro diminui de uma unidade. Dessa forma, o prazo das taxas de nossa base muda dia a dia. Evidentemente, como é de praxe na literatura de renda fixa, poderíamos interpolar diariamente para prazos fixos os dados obtidos. Optamos por uma abordagem diferente para evitar que erros de interpolação contaminassem os resultados.

\section{O MODELO DL}

O modelo DL é uma variante do modelo paramétrico NS no qual a taxa de juros à vista na data $t$ para o prazo $\tau$, denotada por $y_{t}(\tau)$, tem a seguinte forma funcional:

$$
y_{t}(\tau)=\beta_{1 t}+\beta_{2 t}\left(\frac{1-e^{-\lambda_{t} \tau}}{\lambda_{t} \tau}\right)+\beta_{3 t}\left(\frac{1-e^{-\lambda_{t} \tau}}{\lambda_{t} \tau}-e^{-\lambda_{t} \tau}\right)
$$

O parâmetro $\lambda_{t}$ controla a taxa de decaimento exponencial da inclinação e a curvatura da curva de juros. Valores pequenos de $\lambda_{t}$ provocam uma redução suave nos coeficientes exponenciais e servem para ajustar bem a curva em prazos longos. Por outro lado, valores altos de $\lambda_{t}$ resultam em uma redução dos coeficientes exponenciais mais acentuada e servem para ajustar a curva em prazos mais curtos.

Os coeficientes $\beta_{1 t}, \beta_{2 t}$ e $\beta_{3 t}$ podem ser interpretados como fatores latentes dinâmicos. O loading do primeiro fator é constante e igual a 1, portanto $\beta_{1 t}$ pode ser interpretado como o fator de longo prazo. O loading de $\beta_{2 t}$ é representado por uma função que assume o valor limite igual a 1 para a maturidade tendendo a zero e decai monotonicamente para zero com o aumento da maturidade. Assim, podemos interpretar o segundo fator como o fator de curto prazo. Finalmente, o loading de $\beta_{3 t}$ assume valor limite igual a zero para a maturidade próxima de zero, cresce atingindo seu máximo e depois decai para zero novamente, mas de uma forma mais lenta do que o segundo loading. Dessa forma o terceiro fator representa o fator de médio prazo. A Figura A-1 apresenta os loadings desses três fatores.

Alternativamente, os fatores latentes podem ser interpretados como os três movimentos básicos da curva de juros. ${ }^{9}$ Um aumento em $\beta_{1 t}$ provoca um aumento igual nas taxas para todas as maturidades, logo podemos interpretar esse fator como o nível da curva. Um aumento em $\beta_{2 t}$ gera uma variação maior nas taxas longas que nas curtas. Portanto, podemos interpretar esse fator como a inclinação. Um aumento em $\beta_{3 t}$ tem pouco efeito nas taxas curtas e longas, mas afeta as taxas médias. Logo podemos interpretar esse fator como a curvatura.

Os parâmetros $\beta_{1 t}, \beta_{2 t}, \beta_{3 t}$ e $\lambda_{t}$ podem ser estimados em cada dia $t$ via um procedimento de mínimos quadrados não lineraes. Diebold e Li (2006) propõem, entretanto, uma abordagem mais simples.

\footnotetext{
${ }^{6}$ Dia de saque é um dia útil para fins de operações praticadas no mercado financeiro, conforme estabelecido pelo Conselho Monetário Nacional.

${ }^{7}$ Taxa DI é a taxa média de depósitos interfinanceiros de 1 dia calculada pela Central de Custódia e de Liquidação Financeira de Títulos (Cetip).

${ }^{8}$ Para maiores detalhes sobre o DI futuro, veja as especificações do contrato desse derivativo disponível no site http://www.bmf.com.br.

${ }^{9}$ Para uma descrição detalhada dos movimentos da curva de juros veja Litterman e Scheinkman (1991).
} 
Primeiramente fixa-se o valor do parâmetro de decaimento $\left(\lambda_{t}=\lambda\right.$ para todo $\left.t\right)$. Em seguida calcula-se o valor dos loadings para esse valor prefixado de $\lambda_{t}$. Por fim, encontram-se os fatores latentes em cada dia $t$ por mínimos quadrados ordinários tomando os loadings conhecidos como regressores. Diebold e Li (2006) fixam o parâmetro de decaimento de tal forma que o máximo do loading do fator de prazo médio coincida com o máximo observado da curvatura. ${ }^{10}$ Seja $\bar{\tau}$ a maturidade onde ocorre o máximo observado da curvatura, então o parâmetro de decaimento deve satisfazer a:

$$
1+\lambda \bar{\tau}+(\lambda \bar{\tau})^{2}=e^{\lambda \bar{\tau}}
$$

Obtida uma série temporal de fatores latentes, a previsão da taxa de prazo $\tau$ na data $t+h$ feita com as informações disponíveis em $t$ é

$$
\hat{y}_{t+h / t}(\tau)=\hat{\beta}_{1, t+h / t}+\hat{\beta}_{2, t+h / t}\left(\frac{1-e^{-\lambda \tau}}{\lambda \tau}\right)+\hat{\beta}_{3, t+h / t}\left(\frac{1-e^{-\lambda \tau}}{\lambda \tau}-e^{-\lambda \tau}\right)
$$

onde

$$
\hat{\beta}_{i, t+h / t}=\hat{c}_{i}+\hat{\gamma}_{i} \hat{\beta}_{i t}, \quad i=1,2,3
$$

com os parâmetros $\hat{c}_{i} \mathrm{e} \hat{\gamma}_{i}$ estimados através de um processo autoregressivo de ordem 1 na série temporal do fator $\beta_{i}$ disponível até $t$.

A principal contribuição deste trabalho é a análise de quatro técnicas diferentes de escolha do parâmetro de decaimento. Assim como Diebold e Li (2006) nós fixamos $\lambda_{t}=\lambda$ para todo $t$. A inovação está na forma de obtenção do valor de $\lambda$. Em outras palavras, nós testamos a robustez do modelo DL em relação a critérios de escolha do parâmetro de decaimento.

Em todas as quatro técnicas, a pesquisa dos lambdas ótimos foi feita considerado-se os dados dos 400 primeiros dias da amostra (de 04/11/2004 a 14/06/2006). Os dados do período que abrange os dias 401 a 534 (de 16/06/2006 a 28/12/2006) foram utilizados para testar a qualidade da previsão gerada por cada técnica.

Na primeira técnica, nós escolhemos o lambda tal que a soma dos quadrados dos erros de ajuste diários nos 400 primeiros dias da amostra fosse a menor possível. O ajuste da curva em cada dia foi obtido através de um procedimento de mínimos quadrados ordinários. Vamos chamar essa técnica de critério 1 de estimação do lambda.

Nas outras três técnicas nós dividimos a amostra em duas sub-bases. A primeira compreende os dias 1 até 300 e a segunda os dias 301 até 400. Em seguida nós determinamos o lambda que minimiza o erro médio quadrático para previsões 1 dia a frente, 1 semana (5 dias úteis) a frente e 1 mês (21 dias úteis) a frente no período da segunda sub-base, feitas estas previsões de acordo com a Equação 3. Assim obtemos três lambdas ótimos. O primeiro deles minimiza o erro médio quadrático de previsão 1 dia a frente no período compreendido entre o dia 301 e 400 da amostra. As previsões são feitas impondo um processo $\mathrm{AR}(1)$ nos fatores latentes conforme a Equação 4. Os outros dois lambdas têm interpretação semelhante. Vamos chamar essas 3 técnicas de critérios 2, 3 e 4 de estimação do lambda.

Os lambdas ótimos obtidos por cada uma dessas técnicas estão indicados na Tabela 1.

\section{ANÁLISE DOS RESULTADOS}

Passemos agora a análise dos resultados obtidos. As Figuras A-2, A-3 e A-4 apresentam as séries temporais dos fatores latentes nível, inclinação e curvatura, respectivamente, estimados por todos os quatro critérios de escolha do lambda. Observe que as séries temporais dos fatores de longo prazo $\left(\beta_{1}\right)$ apresentam uma inversão próxima a transição dentro da amostra (até o dia 400) para fora da amostra (dia

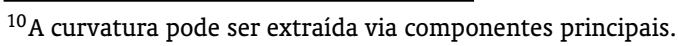


Tabela 1 - Lambdas ótimos estimados por quatro critérios diferentes

\begin{tabular}{|c|c|c|}
\hline Critério & Descrição & Lambda \\
\hline 1 & Ajuste & 1,8610 \\
\hline 2 & Previsão 1 dia & 2,5831 \\
\hline 3 & Previsão 1 semana & 5,5288 \\
\hline 4 & Previsão 1 mês & 14,8119 \\
\hline
\end{tabular}

Essa tabela apresenta os lambdas estimados segundo quatro critérios diferentes. O critério ajuste corresponde a escolher o lambda que minimiza o erro de ajuste no período compreendido entre o dia 1 e o dia 400. Previsão 1 dia minimiza o erro de previsão 1 dia a frente no período entre os dias 301 e 400. Previsão 1 semana minimiza o erro de previsão 1 semana a frente no período entre os dias 301 e 400. Previsão 1 mês minimiza o erro de previsão 1 mês a frente no período entre os dias $301 \mathrm{e}$ 400

401 até 534): dentro da amostra o $\beta_{1}$ estimado pelo critério 1 é o menor enquanto o $\beta_{1}$ estimado pelo critério 4 é o maior; fora da amostra esses papéis são trocados. Isso pode explicar a melhor capacidade preditiva do critério 4 para prazos mais longos visto que no período de análise a economia brasileira passou por um momento de redução da taxa de juros básica. Os fatores latentes correspondentes as taxas de curto $\left(\beta_{2}\right)$ e médio $\left(\beta_{3}\right)$ prazos apresentam um comportamento bem parecido quando estimados pelos critérios 1,2 e 3 e um certo distúrbio quando estimado pelo critério 4 . A dificuldade para previsões de curto prazo demonstrada pelo critério 4 pode estar relacinada com essa pertubação na inclinação e no nível.

As Tabelas 2 e 3 apresentam as médias dos erros médios quadráticos das previsões de 1 dia, 1 semana e 1 mês a frente de todas as taxas para os quatro critérios propostos de escolha do lambda ótimo. Os resultados estão em pontos base. Na Tabela 2 os erros médios foram calculados para o mesmo período em que os lambdas ótimos foram determinados pelos critérios 2, 3 e 4, ou seja, entre os dias 301 e 400. Já na Tabela 3 os erros reportados referem-se ao período fora da amostra, isto é, entre os dias 401 e 534. Todas as previsões foram feitas com os dados até o dia anterior ao dia considerado, reestimando dia a dia os processos autoregressivos descritos pela Equação 4.

Tabela 2 - Média dos erros médios quadráticos de previsão de todas as taxas entre os dias 301 e 400

\begin{tabular}{|c|c|c|c|}
\hline Critério & Previsão 1 dia & Previsão 1 semana & Previsão 1 mês \\
\hline 1 & 19,54 & 45,35 & 143,43 \\
\hline 2 & 18,78 & 41,29 & 132,73 \\
\hline 3 & 21,82 & 36,65 & 97,55 \\
\hline 4 & 36,06 & 48,50 & 70,10 \\
\hline
\end{tabular}

Essa tabela apresenta as médias dos erros médios quadráticos em pontos base de todas as taxas para previsões 1 dia, 1 semana e 1 mês a frente. Os erros foram calculados dentro da amostra entre os dias 301 e 400, período de determinação do lambda ótimo para os critérios 2,3 e 4

A Tabela 2 mostra que os menores erros médios quadráticos dentro da amostra para os três prazos de previsão são aqueles obtidos usando os lambdas ótimos correspondentes, o que não apresenta novidade alguma visto que isso é a condição imposta pelos critérios 2,3 e 4 de escolha do parâmetro de decaimento. Por outro lado, observando a Tabela 3 vemos que esse resultado continua valendo para as 
Tabela 3 - Média dos erros médios quadráticos de previsão de todas as taxas entre os dias 401 e 534

\begin{tabular}{|c|c|c|c|}
\hline Critério & Previsão 1 dia & Previsão 1 semana & Previsão 1 mês \\
\hline 1 & 11,18 & 33,67 & 106,22 \\
\hline 2 & 10,57 & 31,99 & 106,34 \\
\hline 3 & 15,20 & 30,73 & 86,64 \\
\hline 4 & 24,10 & 39,14 & 73,10 \\
\hline
\end{tabular}

Essa tabela apresenta as médias dos erros médios quadráticos em pontos base de todas as taxas para previsões 1 dia, 1 semana e 1 mês a frente. Os erros foram calculados fora da amostra entre os dias 401 e 534

previsões fora da amostra. Ainda da análise dessas duas tabelas, notamos que o critério 1 (ajuste) não é ótimo para nenhum horizonte de previsão sendo dominado pelo critério $2 .{ }^{11}$

Descompactando os dados das Tabelas 2 e 3 podemos observar como variam os erros de previsão ao longo de toda a curva de juros. As Tabelas 4, 5 e 6 mostram o erro médio quadrático de previsão da taxa de contratos DI futuro de prazos curtíssimo, curto, médio-curto, médio, médio-longo e longo ${ }^{12}$ para os horizontes de previsão de 1 dia, 1 semana e 1 mês, respectivamente.

Tabela 4 - Erros médios quadráticos de previsão 1 dia a frente fora da amostra para DI's futuro de diferentes prazos

\begin{tabular}{|c|c|c|c|c|c|c|}
\hline Critério & Curtíssimo & Curto & $\begin{array}{c}\text { Médio } \\
\text { curto }\end{array}$ & Médio & $\begin{array}{c}\text { Médio } \\
\text { longo }\end{array}$ & Longo \\
\hline 1 & $\mathbf{1 0 , 3 6}$ & 8,58 & 11,01 & 10,60 & $\mathbf{1 0 , 4 9}$ & $\mathbf{1 0 , 8 5}$ \\
\hline 2 & 13,43 & 6,10 & $\mathbf{6 , 6 5}$ & $\mathbf{1 0 , 1 8}$ & 11,60 & 11,72 \\
\hline 3 & 24,65 & $\mathbf{5 , 6 8}$ & 10,29 & 22,21 & 19,15 & 12,53 \\
\hline 4 & 20,79 & 23,13 & 36,94 & 34,93 & 22,26 & 12,70 \\
\hline
\end{tabular}

Essa tabela apresenta os erros médios quadráticos em pontos base para previsões 1 dia a frente dos DI's futuro de prazo curtíssimo (primeiro vencimento), curto (terceiro vencimento), médio-curto (quinto vencimento), médio (sétimo vencimento), médio-longo (nono vencimento) e longo (décimo segundo vencimento). Os erros foram calculados fora da amostra entre os dias 401 e 534. Em destaque o menor erro para cada prazo

Conclusões interessantes podem ser feitas a partir da análise do erro médio quadrático ao longo de toda a curva. Primeiramente, observe que o critério 1 apresenta um bom poder de previsão da taxa curtíssima para horizontes de 1 dia e 1 semana a frente. Além disso, esse critério tem a menor dispersão de erros ao longo da curva para todos os horizontes de previsão. O critério 1 não favorece uma taxa em particular em detrimento de outra. Em outras palavras, ele tem a melhor capacidade de prever a curva de juros como um todo. O critério 2 possui um bom desempenho na parte curta e média da curva para horizontes curtos de previsão. No entanto, a capacidade preditiva do critério 2 para taxas de prazo curtíssimo é fraca. O critério 3 é o dominante para todos os horizontes de previsão quando se pretende capturar a parte curta da curva, porém, assim como o critério 2, ele é deficiente na previsão de curtíssimo prazo. Por fim, o critério 4 apresenta boa qualidade de previsão para taxas longas e também para o horizonte de 1 mês a frente.

Para confirmar estatisticamente se existe ou não diferença entre as previsões geradas pelos quatro critérios de escolha de lambda, nós aplicamos aos resultados obtidos o teste de comparação de previsões de Diebold e Mariano (1995). Fixamos o termo de comparação como sendo o critério 1 de

${ }^{11}$ Melhor entender essa dominância em um sentido fraco, visto que para previsão 1 mês a frente os critérios 1 e 2 apresentam praticamente o mesmo desempenho médio.

${ }^{12}$ Estes prazos correspondem ao primeiro, terceiro, quinto, sétimo, nono e décimo segundo vencimento em cada dia. Em média, esses DI’s vencem em 13, 55, 129, 255, 391 e 610 dias úteis. 
Tabela 5 - Erros médios quadráticos de previsão 5 dias a frente fora da amostra para DI's futuro de diferentes prazos

\begin{tabular}{|c|c|c|c|c|c|c|}
\hline Critério & Curtíssimo & Curto & $\begin{array}{c}\text { Médio } \\
\text { curto }\end{array}$ & Médio & $\begin{array}{c}\text { Médio } \\
\text { longo }\end{array}$ & Longo \\
\hline 1 & 34,84 & 34,02 & 35,39 & 34,74 & $\mathbf{3 3 , 6 7}$ & 33,65 \\
\hline 2 & 38,14 & 29,95 & 29,55 & $\mathbf{3 2 , 7 7}$ & 33,85 & 33,55 \\
\hline 3 & 46,03 & $\mathbf{2 0 , 0 5}$ & $\mathbf{2 8 , 4 5}$ & 39,88 & 37,08 & 28,95 \\
\hline 4 & $\mathbf{3 4 , 7 0}$ & 51,43 & 56,71 & 50,88 & 37,87 & $\mathbf{2 3 , 7 2}$ \\
\hline
\end{tabular}

Essa tabela apresenta os erros médios quadráticos em pontos base para previsões 5 dias a frente dos DI's futuro de prazo curtíssimo (primeiro vencimento), curto (terceiro vencimento), médio-curto (quinto vencimento), médio (sétimo vencimento), médio-longo (nono vencimento) e longo (décimo segundo vencimento). Os erros foram calculados fora da amostra entre os dias 401 e 534. Em destaque o menor erro para cada prazo

Tabela 6 - Erros médios quadráticos de previsão 21 dias a frente fora da amostra para DI's futuro de diferentes prazos

\begin{tabular}{|c|c|c|c|c|c|c|}
\hline Critério & Curtíssimo & Curto & $\begin{array}{c}\text { Médio } \\
\text { curto }\end{array}$ & Médio & $\begin{array}{c}\text { Médio } \\
\text { longo }\end{array}$ & Longo \\
\hline 1 & 119,73 & 116,77 & 114,98 & 110,92 & 107,38 & 103,18 \\
\hline 2 & 128,91 & 115,56 & 110,11 & 109,07 & 108,26 & 104,54 \\
\hline 3 & 113,50 & $\mathbf{8 2 , 6 6}$ & $\mathbf{8 6 , 1 9}$ & 96,65 & 95,17 & 85,44 \\
\hline 4 & $\mathbf{7 2 , 9 9}$ & 83,90 & 90,05 & $\mathbf{8 8 , 3 8}$ & $\mathbf{7 8 , 6 2}$ & $\mathbf{6 3 , 0 1}$ \\
\hline
\end{tabular}

Essa tabela apresenta os erros médios quadráticos em pontos base para previsões 21 dias a frente dos DI's futuro de prazo curtíssimo (primeiro vencimento), curto (terceiro vencimento), médio-curto (quinto vencimento), médio (sétimo vencimento), médio-longo (nono vencimento) e longo (décimo segundo vencimento). Os erros foram calculados fora da amostra entre os dias 401 e 534. Em destaque o menor erro para cada prazo

escolha do lambda. A Tabela 7 apresenta a estatística S1 para uma função de perda quadrática tomando como competidor do termo de comparação os critérios 2, 3 e 4 . Entradas positivas indicam dominância do benchmark e negativas o contrário, sendo que valores absolutos maiores que 1,96 representam significância de $95 \%$. Por um lado, a estatística S1 corrobora estatisticamente algumas conclusões do parágrafo anterior, como por exemplo, o bom desempenho na parte curta e média da curva dos critérios 2 e 3, especialmente para os horizontes de previsão de 1 dia e 1 semana a frente. Por outro lado, ela enfraquece outras conclusões, como por exemplo, a dominância do critério 4 para previsões 1 mês a frente.

\section{CONCLUSÃO}

O objetivo desse trabalho foi avaliar o poder de previsão do modelo DL usando quatro métodos diferentes de escolha do parâmetro que controla a inclinação e a curvatura da curva de juros. Os resultados mostram que a regra ótima de escolha desse parâmetro depende do horizonte de previsão e da taxa que se pretende prever.

Em média, o critério que seleciona o parâmetro de controle da curvatura que minimiza os erros de ajuste dentro da amostra é o superior. Ele não favorece nem discrimina um vencimento de DI ou um horizonte de previsão específicos. Quando o objetivo é analisar fatos estilizados do mercado ou propriedades econômicas relacionadas à curva de juros, esse critério é o recomendado. No entanto, se o propósito é realizar uma previsão ou simular um cenário para o preço de um ativo, outros critérios podem ser mais úteis. Por exemplo, para realizar previsões 1 dia a frente de taxas de prazo em torno de 
Tabela 7 - Estatística S1 de significância entre as diferenças de previsões fora da amostra: Teste de Diebold e Mariano (1995)

\begin{tabular}{|c|c|c|c|c|c|c|}
\hline Prazo & Curtíssimo & Curto & $\begin{array}{c}\text { Médio } \\
\text { curto }\end{array}$ & Médio & $\begin{array}{c}\text { Médio } \\
\text { longo }\end{array}$ & Longo \\
\hline Critério & \multicolumn{7}{|c|}{ Previsão 1 dia a frente } \\
\hline 2 & 7,60 & $-17,36$ & $-18,48$ & $-3,41$ & 4,30 & 3,48 \\
\hline 3 & 5,24 & $-6,26$ & $-1,55$ & 12,15 & 10,97 & 3,34 \\
\hline 4 & 2,53 & 5,82 & 7,75 & 10,47 & 10,99 & 2,13 \\
\hline \hline Critério & 3,17 & $-4,17$ & $-5,70$ & $-2,27$ & 0,24 & $-0,01$ \\
\hline 2 & 2,41 & $-5,74$ & $-2,61$ & 1,67 & 1,37 & $-1,92$ \\
\hline 3 & $-0,022$ & 2,00 & 2,33 & 2,45 & 1,06 & $-3,31$ \\
\hline 4 & \multicolumn{7}{|c|}{ Previsão 1 mês a frente } \\
\hline \hline Critério & 1,90 & $-0,23$ & $-0,96$ & $-0,43$ & 0,23 & 0,31 \\
\hline 2 & $-0,33$ & $-2,03$ & $-1,81$ & $-0,92$ & $-0,78$ & $-1,03$ \\
\hline 3 & $-1,56$ & $-1,23$ & $-0,95$ & $-0,91$ & $-1,22$ & $-1,67$ \\
\hline 4 &
\end{tabular}

Essa tabela apresenta a estatística S1 de Diebold e Mariano (1995) para previsões 1 dia a frente, 1 semana a frente e 1 mês a frente fora da amostra (dias 401 a 534). O termo de comparação é o critério 1 de escolha do lambda e a função de perda é o erro médio quadrático. Entradas positivas representam dominância de previsão do critério 1. Valores absolutos grandes para S1 indicam alta probabilidade de rejeitar a hipótese nula (diferença entre os erros médios quadráticos desprezível). Valores absolutos maiores que 1,96 correspondem a uma rejeição da hipótese nula a um nível de confiança de $95 \%$

3 meses, é recomendado que o parâmetro de curvatura seja escolhido de forma a minimizar os erros de previsão 1 dia a frente em uma sub-base dentro da amostra.

Os resultados mostram ainda que o modelo DL carece de robutez, visto que as conclusões são muito sensíveis ao valor do parâmetro de curvatura. Muito provavelmente, esse problema está relacionado com a dificuldade de capturar a dinâmica do prêmio de risco via a combinação de modelos paramétricos com processos autoregressivos de ordem 1 (veja Almeida e Vicente (2008)). Nesse sentido, extensões do modelo DL original como as propostas por Almeida et alii (2007) e Laurini e Hotta (2007) são promissoras.

\section{BIBLIOGRAFIA}

Almeida, C., Duarte, A., \& Fernandes, C. (2003). A generalization of principal components analysis for non-observable term structures in emerging markets. International Journal of Theoretical and Applied Finance, 6(8):885-903.

Almeida, C., Gomes, R., Leite, A., \& Vicente, J. (2007). Does curvature enhance forecasting? Technical Report 155, Banco Central do Brasil.

Almeida, C. \& Vicente, J. (2008). The role of no-arbitrage on forecasting: Lessons from a parametric term structure model. Journal of Banking \& Finance (a sair).

Dai, Q. \& Singleton, K. (2000). Specification analysis of affine term structure models. Journal of Finance, 55(5):1943-1978.

De Pooter, M. (2007). Examining the Nelson-Siegel class of term structure models: In-sample fit versus out-of-sample forecasting performance. Technical Report 043/4, Tinbergen Institute. 
Diebold, F. \& Li, C. (2006). Forecasting the term structure of government bond yields. Journal of Econometrics, 130(2):337-364.

Diebold, F. \& Mariano, R. (1995). Comparing predictive accuracy. Journal of Business and Economic Statistics, 13:253-265.

Duffee, G. (2002). Term premia and interest rate forecasts in affine models. Journal of Finance, 57(1):405-443.

Filipovic, D. \& Sharef, E. (2004). Conditions for consistent exponential-polynomial forward rate processes with multiple nontrivial factors. International Journal of Theoretical and Applied Finance, 7(6):685-700.

Huse, C. (2007). Term structure modelling with observable state variables. Technical report, London School of Economics.

Koopman, S., Mallee, M., \& Van der Wel, M. (2008). Analyzing the term structure of interest rates using the dynamic Nelson-Siegel model with time-varying parameters. Journal of Business and Economic Statistics (a sair).

Laurini, M. \& Hotta, L. (2007). Extensões bayesianas do modelo de estrutura a termo de Diebold-Li. Technical Report 40, IBMEC.

Lima, E., Luduvice, F., \& Tabak, B. (2006). Forecasting interest rates: An application for Brazil. Technical Report 120, Banco Central do Brasil.

Litterman, R. \& Scheinkman, J. (1991). Common factors affecting bond returns. The Journal of Fixed Income, 1(1):54-61.

Nelson, C. \& Siegel, A. (1987). Parsimonious modeling of yield curves. The Journal of Business, 60(4):473-489.

Svensson, L. (1994). Monetary policy with flexible exchange rates and forward interest rates as indicators. Technical Report 4633, National Bureau of Economic Research.

Varga, G. (2005). Teste de modelos estatísticos para a estrutura a termo no brasil. XXIX Encontro da Associação Nacional de Pós-Graduação e Pesquisa em Administração.

Varga, G. (2007). Brazilian (local) term structure forecast in a factor model. VII Encontro Brasileiro de Finanças.

Vicente, J. \& Tabak, B. (2008). Forecasting bond yields in the brazilian fixed income market. International Journal of Forecasting, 24(3):490-497. 


\section{A. FIGURAS}

Figura A-1 - Loadings do modelo DL. A figura apresenta os loadings do modelo DL correspondente aos fatores nível $\left(\beta_{1}\right)$, inclinação $\left(\beta_{2}\right)$ e curvatura $\left(\beta_{3}\right)$ considerando $\lambda=5,5288$ (fixo).

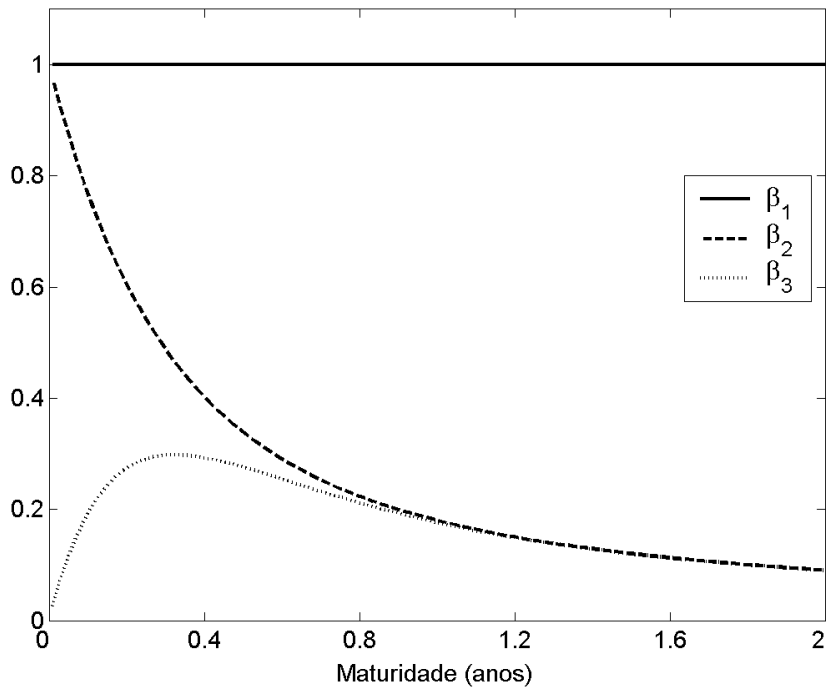


Figura A-2 - Série temporal do fator latente nível para cada um dos critérios de escolha de lambda. A figura apresenta a série temporal do fator latente nível para cada um dos critérios de escolha do parâmetro de decaimento ao longo dos 534 dias analisados nesse estudo.

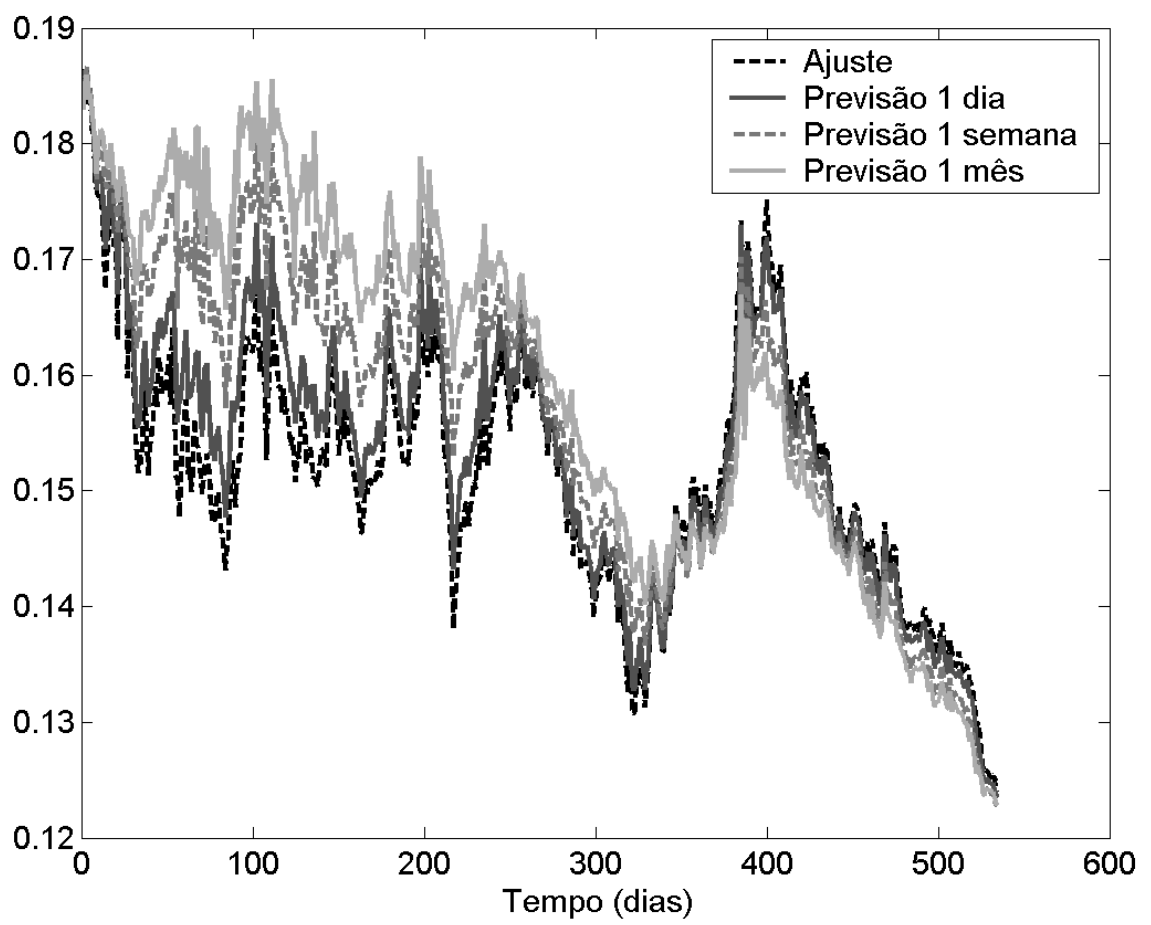


Figura A-3 - Série temporal do fator latente inclinação para cada um dos critérios de escolha de lambda. Essa figura apresenta a série temporal do fator latente inclinação para cada um dos critérios de escolha do parâmetro de decaimento ao longo dos 534 dias analisados nesse estudo.

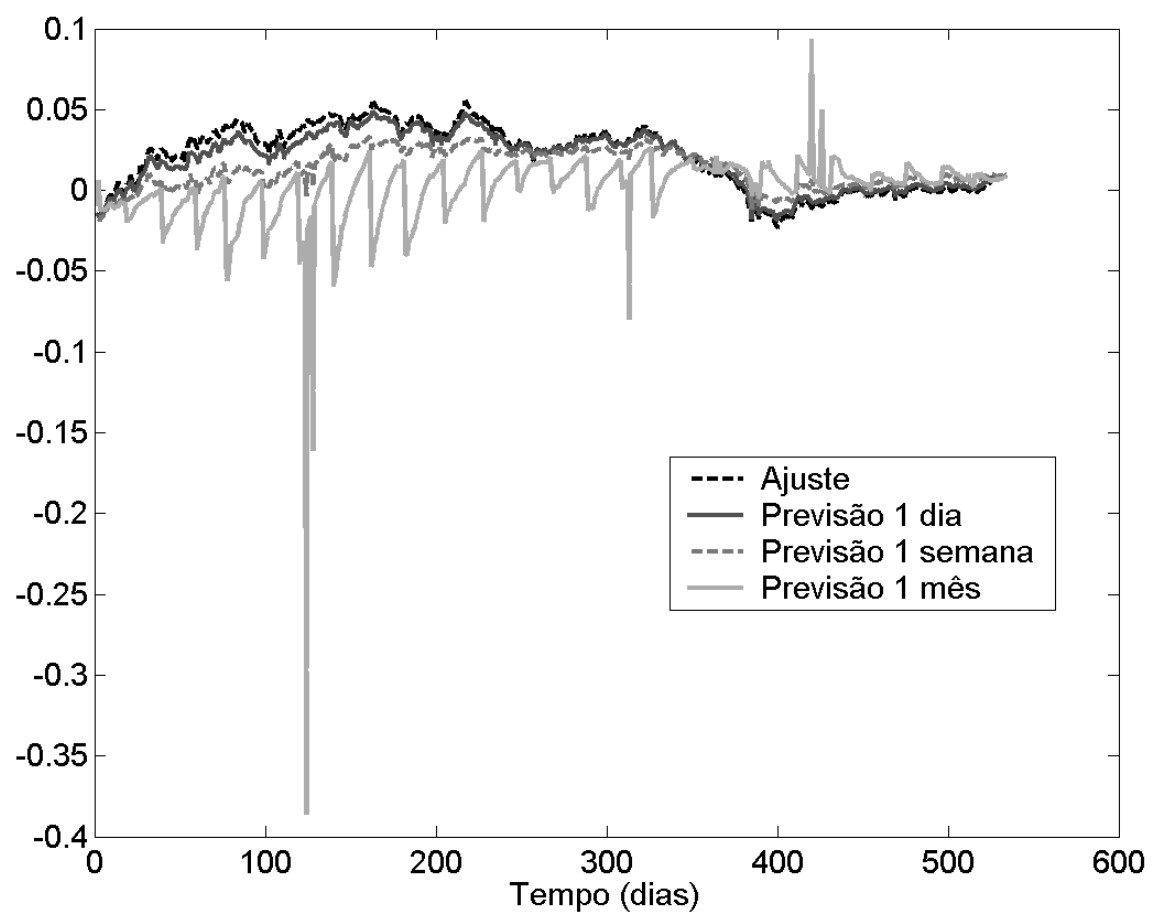


Figura A-4 - Série temporal do fator latente curvatura para cada um dos critérios de escolha de lambda. Essa figura apresenta a série temporal do fator latente curvatura para cada um dos critérios de escolha do parâmetro de decaimento ao longo dos 534 dias analisados nesse estudo.

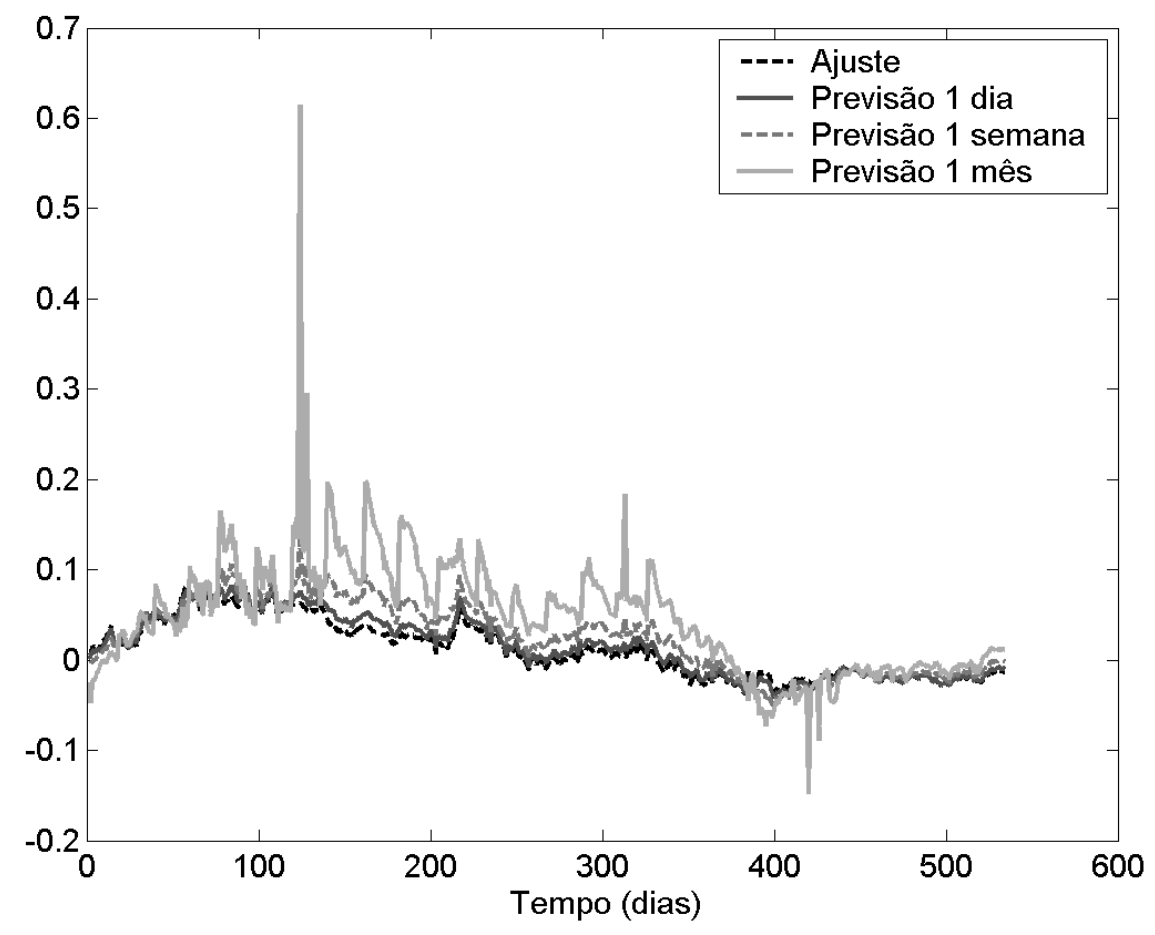

